\title{
Reversible Cerebral Vasoconstriction Syndrome, Part 2: Diagnostic Work-Up, Imaging Evaluation, and Differential Diagnosis
}

\author{
T.R. Miller, R. Shivashankar, M. Mossa-Basha, and D. Gandhi
}

\begin{abstract}
SUMMARY: The diagnostic evaluation of a patient with reversible cerebral vasoconstriction syndrome integrates clinical, laboratory, and radiologic findings. Imaging plays an important role by confirming the presence of cerebral vasoconstriction; monitoring potential complications such as ischemic stroke; and suggesting alternative diagnoses, including CNS vasculitis and aneurysmal subarachnoid hemorrhage. Noninvasive vascular imaging, including transcranial Doppler sonography and MR angiography, has played an increasingly important role in this regard, though conventional angiography remains the criterion standard for the evaluation of cerebral artery vasoconstriction. Newer imaging techniques, including high-resolution vessel wall imaging, may help in the future to better discriminate reversible cerebral vasoconstriction syndrome from primary angiitis of the CNS, an important clinical distinction.
\end{abstract}

ABBREVIATIONS: PACNS = primary angiitis of the CNS; RCVS = reversible cerebral vasoconstriction syndrome; TCD = transcranial Doppler sonography; VWI = vessel wall imaging

$\mathbf{R}$ eversible cerebral vasoconstriction syndrome (RCVS) is a clinical and radiologic syndrome characterized by the hyperacute onset of severe headache and reversible segmental vasoconstriction of the cerebral arterial vasculature. ${ }^{1-5}$ In the first part of this review, we discussed the historical background, possible pathogenesis, and clinical features of RCVS. In this second part, we will explore the diagnostic work-up of suspected cases of RCVS, with an emphasis on the role of imaging in the diagnosis of the entity and monitoring potential complications. This includes high-resolution MR vessel wall imaging (VWI), a new technique that may help differentiate RCVS from alternative diagnoses by characterizing pathologic changes in the wall of affected cerebral arteries. Finally, we will discuss how to integrate both clinical and radiographic features in suspected cases of RCVS to formulate a tailored differential diagnosis. Special emphasis will be placed on differentiating RCVS from aneurysmal subarachnoid hemorrhage and primary angiitis of the CNS (PACNS).

From the Department of Diagnostic Radiology (T.R.M., R.S., D.G.), Section of Neuroradiology, University of Maryland Medical Center, Baltimore, Maryland; and Department of Diagnostic Radiology (M.M.-B.), Section of Neuroradiology, University of Washington, Seattle, Washington.

Please address correspondence to Dheeraj Gandhi, MD, University of Maryland Medical Center, Department of Diagnostic Radiology, Room N2W78, 22 South Greene St, Baltimore, MD 21201; e-mail: Dgandhi@umm.edu

-- Indicates open access to non-subscribers at www.ajnr.org

http://dx.doi.org/10.3174/ajnr.A4215

\section{Diagnostic Work-Up and Imaging Evaluation}

Appropriate care of patients suspected of having RCVS begins with a prompt diagnostic work-up to exclude alternative diagnoses, such as aneurysmal subarachnoid hemorrhage and PACNS. ${ }^{6}$ This includes obtaining a complete medical history, with particular attention paid to any common RCVS triggers that may be present; detailed physical examination; CSF analysis; and noncontrast head CT to evaluate for intracranial hemorrhage. ${ }^{2,7-9}$ CSF analysis is an important element in the diagnostic work-up of RCVS, which is reflected in its inclusion in the diagnostic criteria of the syndrome. In most cases of RCVS, findings of CSF analysis will be unremarkable, with red and white blood cell counts and protein levels either within normal limits or only mildly elevated. $^{2,7,9-13}$ Finally, findings of other laboratory tests, including serum analysis for markers of inflammation such as erythrocyte sedimentation rate and C-reactive protein, are also usually within normal limits in patients with RCVS. ${ }^{2,9,14}$

The role of neuroimaging in patients with RCVS includes demonstration of cerebral vasoconstriction, evaluation of alternative diagnoses, and monitoring potential complications such as intracranial hemorrhage, vasogenic edema, and ischemic stroke. ${ }^{4,7,9}$ Although conventional angiography has been the criterion standard for evaluation of cerebral vasoconstriction in suspected cases of RCVS, noninvasive imaging modalities such as transcranial Doppler sonography (TCD), CT angiography, and MR angiography are being used with increasing frequency (Table 1). ${ }^{4,5,9,12,15}$ When present, cerebral vasoconstriction involves 
Table 1: Role of imaging modalities in the management of RCVS Role of Imaging Modalities

Transcranial Doppler sonography
Diagnosis of vasoconstriction
Monitoring of vasoconstriction
Noncontrast CT, CT angiography
Detection of cerebral vasoconstriction
Evaluation for complications such as SAH
Provide plausible alternative diagnoses such as cerebral aneurysm
MR imaging, MR angiography
Detection of cerebral vasoconstriction
Evaluation for complications such as ischemic stroke
Provide plausible alternative diagnoses such as cortical vein
thrombosis
Vessel wall imaging
Catheter angiography
Detection of cerebral vasoconstriction, criterion standard
Demonstration of reversibility of vasoconstriction following
intra-arterial vasodilator therapy
Possible treatment with vasodilator or balloon angioplasty

multiple vascular territories and results in a beaded appearance of medium-to-large cerebral arteries with multifocal areas of narrowing interspersed with normal-caliber segments. ${ }^{1,4,7,12,14}$ The severity and distribution of vasoconstriction can fluctuate among examinations, with some areas improving and others worsening. ${ }^{1,2,9,16,17}$ Although the above angiographic findings are highly suggestive of RCVS in the appropriate clinical setting, they remain nonspecific and can be encountered with various other types of CNS vasculopathies and vasculitis. ${ }^{2,14,18-20}$

The initial angiographic evaluation findings in suspected cases of RCVS may be unremarkable in the $4-5$ days following patient presentation. ${ }^{1,2,4,5,21}$ In fact, cerebral vasoconstriction may not be visualized in up to one-third of patients with RCVS during the first week following symptom onset. ${ }^{22}$ As suggested by Ducros and Bousser, ${ }^{2}$ this finding may be due to segmental vasoconstriction in RCVS beginning in small, peripheral arterioles before subsequently proceeding centripetally to involve medium and large cerebral arteries, which are more readily visualized. ${ }^{23}$ If cerebral vasoconstriction is not demonstrated on initial vascular imaging and other diagnoses have been excluded, the patient should be managed as if he or she has possible or probable RCVS. ${ }^{1}$

The following sections further explore the various imaging techniques available for evaluation of suspected cases of RCVS, followed by a more detailed discussion of alternative diagnoses.

\section{Transcranial Doppler Sonography}

TCD has been used to monitor the evolution of vasoconstriction in patients with RCVS by measuring mean and peak blood flow velocities in proximal cerebral arteries around the circle of Willis. ${ }^{9,21,24}$ A prospective study of 67 patients found that $69 \%$ of patients with RCVS had elevated middle cerebral and internal carotid artery velocities, with means of 163 and $148 \mathrm{~cm} / \mathrm{s}$, respectively. ${ }^{12}$ Peak mean cerebral flow velocities were typically present just $>3$ weeks following symptom onset (22 days). Serial TCD examinations may be more sensitive for detecting RCVS-related elevations in cerebral arterial flow than single examinations because TCD findings may be unremarkable early in the course of the syndrome. ${ }^{2}$ Normalization of TCD parameters is typically seen by 12 weeks, paralleling the delay in resolution of cerebral vasoconstriction seen with other vascular imaging modalities such as CT and MR angiography. ${ }^{24,25}$

However, alterations in TCD parameters do not appear to be as profound as those seen in patients with aneurysmal subarachnoid hemorrhage. ${ }^{5,24}$ Only $13 \%$ of patients with RCVS in 1 series fulfilled the TCD diagnostic criteria for mild vasospasm. ${ }^{24}$ TCD may play a role in monitoring potential complications of RCVS. Chen et $\mathrm{al}^{24}$ noted that transcranial color Doppler sonography indicators of cerebral vasoconstriction, including elevated mean flow velocities in the MCA $(>120 \mathrm{~cm} / \mathrm{s})$ and an elevated Lindegaard Index $(>3)$, were associated with an increased risk of developing posterior reversible encephalopathy syndrome.

\section{Noncontrast CT and CT Angiography}

Noncontrast head CT should be the initial examination performed for patients presenting with symptoms suggestive of RCVS, particularly thunderclap headache. Noncontrast CT is an effective way to screen patients for the presence of intracranial hemorrhage, including subarachnoid and intraparenchymal hemorrhage, as well as ischemic stroke. Subarachnoid hemorrhage associated with RCVS is often small in amount and confined to cerebral sulci near the vertex. It may be a subtle finding on noncontrast $\mathrm{CT} .{ }^{10}$ If subarachnoid hemorrhage is present, the distribution of blood, cisternal versus sulcal, can help direct further diagnostic evaluations. Finally, noncontrast CT may demonstrate multifocal infarcts of varying ages, which can suggest the alternative diagnosis of CNS vasculitis.

CT angiography can be used to demonstrate segmental vasoconstriction suggestive of RCVS. ${ }^{8}$ It can also serve to evaluate other potential etiologies of patient symptoms and findings, including cerebral aneurysm, pituitary hemorrhage, arterial dissection, and, occasionally, arterial narrowing and irregularity suggestive of CNS vasculitis. Dual-energy CTA may aid in the diagnosis of cerebral vasoconstriction in suspected cases of RCVS and the evaluation of potential alternative diagnoses such as cerebral aneurysm, by improved bone removal at the skull base. ${ }^{8}$ However, one important drawback of this technique is the increased radiation exposure to the patient. ${ }^{8}$ Finally, CT venography can also be performed with CTA with a slightly delayed scan following contrast administration, potentially allowing the diagnosis of cortical vein and/or dural sinus thrombosis.

\section{Brain MR Imaging and MR Angiography}

Brain MR imaging is often performed in suspected cases of RCVS, and findings can appear normal or demonstrate evidence of complications of the syndrome, such as watershed infarcts or posterior reversible encephalopathy syndrome. ${ }^{10,12,14,16}$ For example, T2 FLAIR-weighted imaging can be used to evaluate for subarachnoid hemorrhage and cerebral edema, while diffusion-weighted imaging is helpful in evaluating for watershed infarcts. Susceptibility-weighted imaging can help evaluate the presence of intracranial hemorrhage. Ischemic infarctions in RCVS are typically watershed in location and bilateral, presumably reflecting impaired cerebral blood flow secondary to severe cerebral vasoconstriction. ${ }^{2,7}$ In addition, MR imaging can also evaluate potential alternative diagnoses, including PACNS, dural sinus thrombosis, 


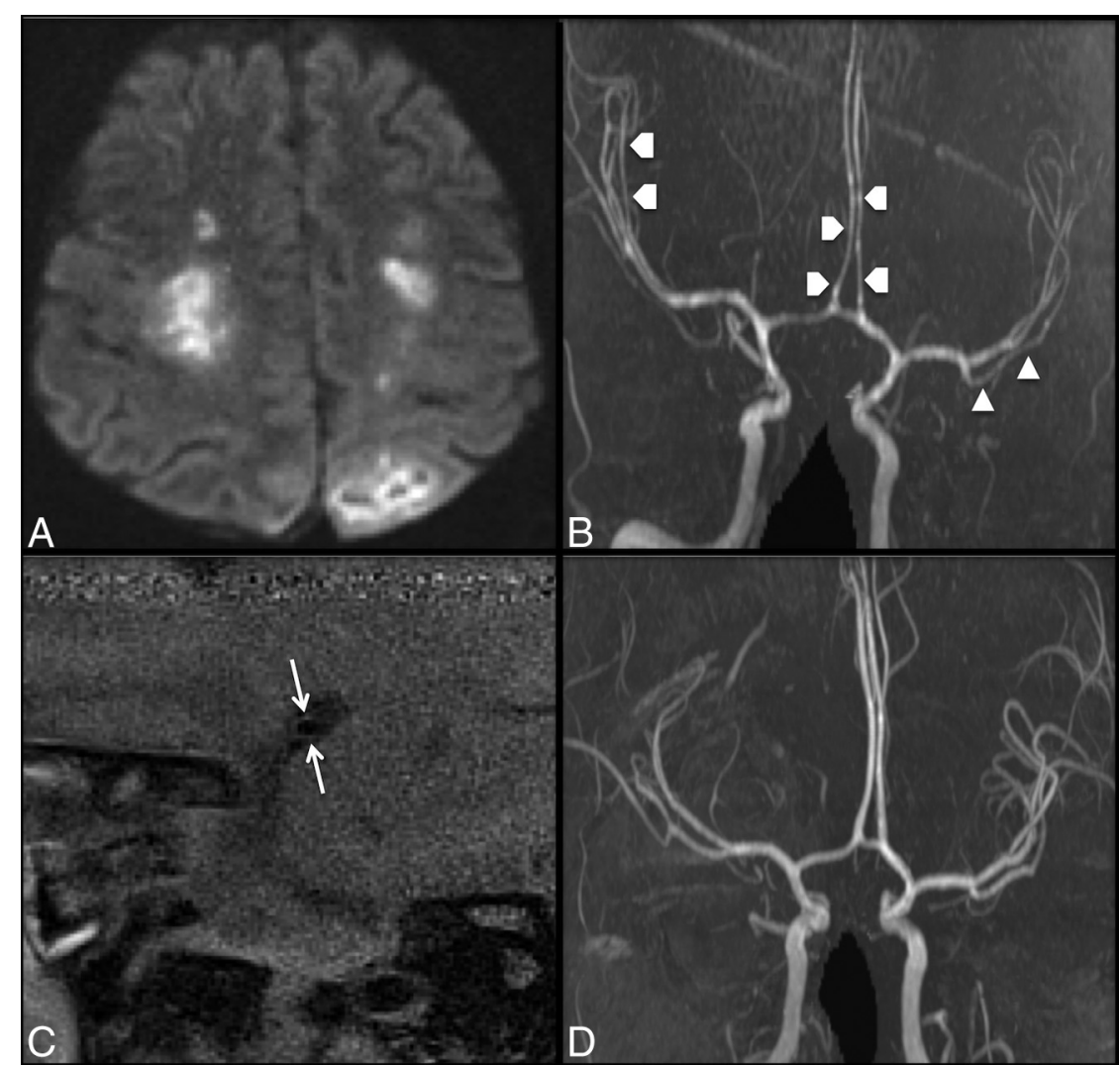

FIG 1. A 55-year-old woman who presented with severe headache and developed left-sided weakness. DWI (A) shows multifocal infarcts involving the centrum semiovale and left posterior parietal lobe. On coronal 3D reformatted TOF MRA $(B)$, there is diffuse narrowing of the bilateral middle and anterior cerebral arteries (white arrowheads). Parasagittal postcontrast T1 high-resolution VWI of the Ml arterial segment of the left MCA (C) shows mild wall thickening and minimal enhancement (similar findings were noted in the right M1 arterial segment, not shown). The patient was diagnosed with RCVS, with subsequent resolution of cerebral vasoconstriction $(D)$. striction peaked in these patients around day 16 following symptom onset and significantly improved in most patients by 1 month. However, the evolution of cerebral vasoconstriction was not uniform, with some cerebral arteries improving on serial scans and others worsening. ${ }^{29}$ They also found that combined segmental vasoconstriction scores in the M1 and P2 arterial segments were most closely associated with the complications of ischemic stroke and posterior reversible encephalopathy syndrome. ${ }^{29}$ One limitation of MRA is the evaluation of small, distal cerebral arteries, which are better evaluated on conventional angiography, given its superior spatial resolution. ${ }^{29}$

\section{Vessel Wall Imaging}

High-resolution MR VWI is a relatively new technique that is being increasingly used in the evaluation of cerebrovascular disease, including CNS vasculitis, RCVS, cerebral aneurysms, Moyamoya disease and syndrome, arterial dissection, and intracranial atherosclerosis. $^{20,30-34}$ This method can use highresolution $2 \mathrm{D}$ or $3 \mathrm{D}$ imaging, frequently with pre- and postcontrast T1 or proton-attenuation sequences. ${ }^{20,32,35-38}$ In addition, high-resolution T2-weighted imaging can be used for multicontrast pituitary apoplexy, cortical vein thrombosis, and arterial dissection.

Hyperintense vessels along cerebral sulci on T2 FLAIR imaging have been noted in patients with RCVS (22\%) and correlate with more severe vasoconstriction as measured by TCD. ${ }^{16,26-28}$ In one study, the presence of hyperintense vessels was associated with a higher risk incidence of ischemic stroke and posterior reversible encephalopathy syndrome. ${ }^{26}$ Hyperintense vessels on T2 FLAIR imaging have previously been described in association with other conditions involving severe cerebral artery stenosis or occlusion, including acute large-vessel ischemic stroke and Moyamoya disease. The hyperintense vessels are thought to represent slow flow in either distal cortical arteries or leptomeningeal anastomotic collaterals. ${ }^{22,27,28}$ However, hyperintense vessels on T2 FLAIR imaging must be differentiated from subarachnoid hemorrhage, which may also be present in patients with RCVS. SWI may be helpful in this regard by identifying the latter. ${ }^{16,22}$

MR angiography is an effective way of diagnosing and monitoring the evolution of RCVS-related vasoconstriction, allowing patients to avoid exposure to ionizing radiation and the small risk of complications associated with conventional angiography. ${ }^{12,29}$ Chen et $\mathrm{al}^{29}$ followed a group of patients with RCVS with serial MRA and showed that the severity of segmental cerebral vasocon- imaging. ${ }^{33,39-41}$ In contradistinction to conventional angiographic imaging techniques that primarily evaluate the blood vessel lumen, such as conventional angiography, this approach provides information regarding the blood vessel wall itself, which is typically only 1-2 mm thick in proximal intracranial vessels. ${ }^{42}$

VWI has been described as black-blood imaging because it results in low signal in the vessel lumen, thereby aiding in the visualization of the blood vessel wall. VWI can be technically challenging to perform however, due to the small caliber and tortuous course of the intracranial arteries, necessitating high spatial resolution and thus high-field-strength magnets. Specific blood vessel wall abnormalities that can be detected by using VWI include vessel wall thickening, which can be further characterized as smooth versus irregular or concentric versus eccentric; and vessel wall enhancement and signal characteristics. ${ }^{20,30,31,43}$

There has been considerable recent interest in using VWI to help differentiate RCVS from CNS vasculitis, which can overlap in clinical and conventional imaging features. ${ }^{20,30}{\text { Mandell et } \mathrm{al}^{30}}^{30}$ used VWI to evaluate a small group of patients presenting with multifocal narrowing of large intracranial arteries, suspicious for vasculitis or RCVS. They found that while patients ultimately diagnosed with both RCVS and CNS vasculitis demonstrated arterial wall thickening, wall enhancement was present only in cases of CNS vasculitis. The authors hypothesized that this finding was 


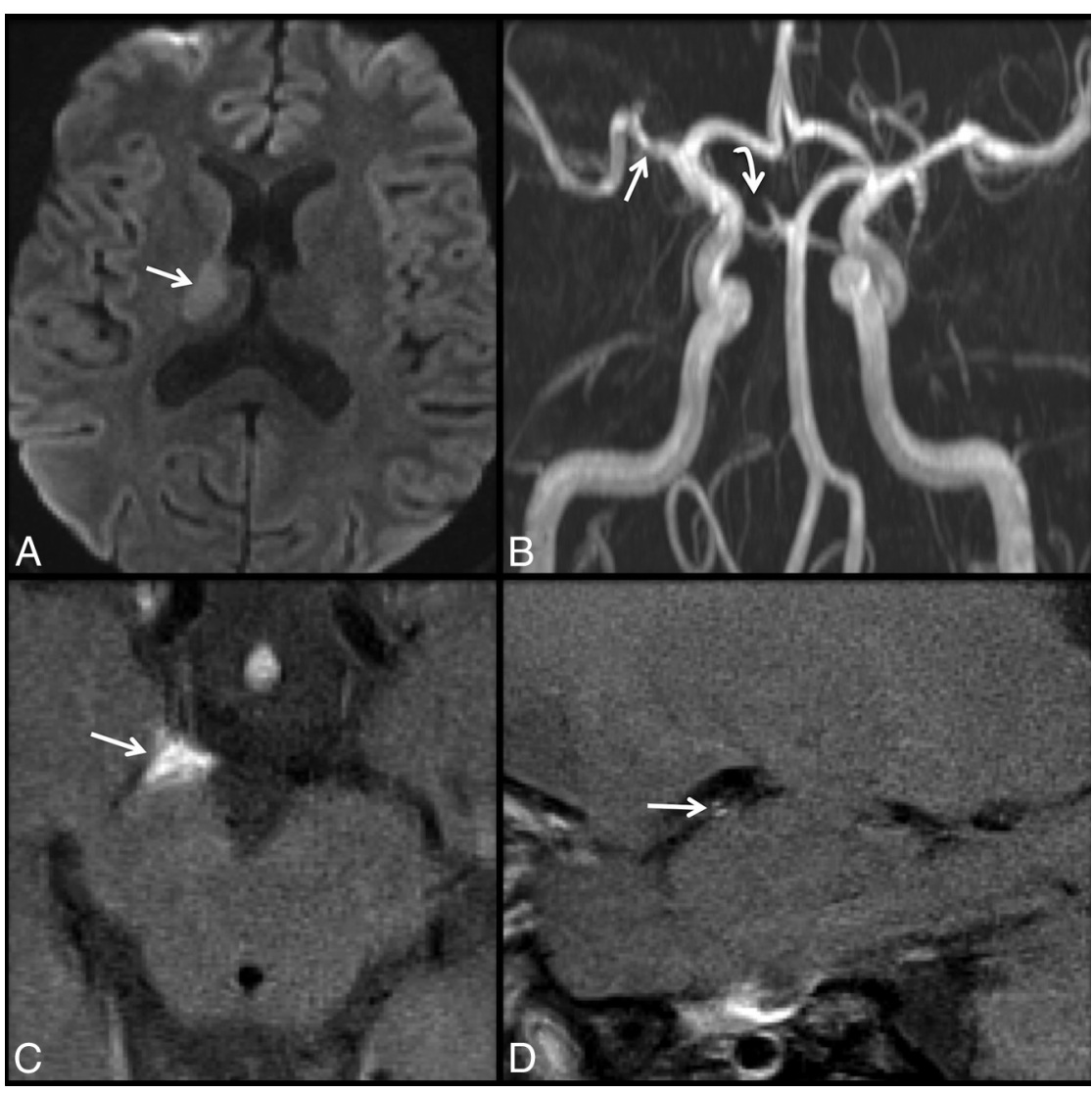

FIG 2. A 35-year-old man with a history of Behçet vasculitis who presented with left-sided weakness. DWI $(A)$ demonstrates an acute infarct involving the right thalamus and posterior limb of the internal capsule (white arrow). Coronal 3D reformat of TOF MRA ( $B$ ) reveals irregularity and narrowing of the $\mathrm{Ml}$ arterial segment of the right MCA (white arrow) and occlusion or high-grade stenosis of the $\mathrm{Pl}$ arterial segment of the right PCA (curved white arrow). On axial T1 postcontrast high-resolution $\mathrm{VWI}(C)$, there is prominent enhancement and enlargement of the right posterior cerebral artery (white arrow). Sagittal TI postcontrast VWI (D) demonstrates typical tram-track, circumferential enhancement of the right MI MCA (white arrow), consistent with vasculitic inflammation.

consistent with pathology results of patients with RCVS who have undergone biopsy-namely, vasoconstriction without an underlying inflammatory vessel wall infiltrate. ${ }^{30}$

A more recent article by Obusez et $\mathrm{al}^{20}$ compared VWI findings in a larger group of patients diagnosed with RCVS and CNS vasculitis ( $n=13$ in each group). They found that 12 of 13 patients diagnosed with CNS vasculitis demonstrated multifocal, short-segment vessel wall thickening, with 9 having concentric and 3 having eccentric wall enhancement (Fig 1). In contradistinction, of the 13 patients diagnosed with RCVS, 10 demonstrated diffuse uniform wall thickening, of which only 4 had associated mild wall enhancement. A minority in each group underwent follow-up VWI, which demonstrated earlier resolution of imaging findings in patients with diagnosed RCVS. These results suggest that VWI may be a useful tool in differentiating RCVS and CNS vasculitis, though further investigation is needed (Fig 2).

\section{Perfusion Imaging}

Perfusion imaging is being increasingly used in the evaluation and monitoring of cerebrovascular diseases such as RCVS and can be performed by using CT or MR imaging techniques. ${ }^{44} \mathrm{CT}$ perfu- sion is performed by repeatedly imaging through the brain during the administration of an iodine contrast bolus. The resulting patient radiation exposure is a potential drawback of this method, particularly in those patients requiring multiple scans. MR perfusion techniques include T1 dynamic contrast-enhanced and dynamic susceptibility contrast MR imaging, the latter performed by rapid, repeat echo-planar imaging of the brain during the passage of a gadolinium contrast bolus, with resulting loss of intra-arterial signal secondary to susceptibility effects from the paramagnetic contrast. ${ }^{44}$ Alternatively, arterial spin-labeling perfusion is a completely noninvasive MR imaging technique that does not require the administration of gadolinium contrast but instead uses an electromagnetic spin inversion to tag water molecules, which then serve as a freely diffusible flow tracer. ${ }^{45}$

On the basis of our own clinical experience and a few isolated case reports, perfusion imaging in RCVS may show multifocal areas of hypoperfusion that often include cerebral watershed zones corresponding to the involved vascular territories (Fig 3). ${ }^{46,47}$ These areas of perfusion abnormality may worsen acutely and, in some instances, progress to watershed infarction as previously discussed. ${ }^{47}$ Changes in cerebral perfusion may correspond to the evolution of arterial vasoconstriction, and this information could potentially be used to track treatment response (eg, vasodilator therapy) and provide physiologic information regarding the effects of individual stenoses. ${ }^{46}$ However, given the relative paucity of published data, further research into the potential role of perfusion imaging in the evaluation and monitoring of RCVS is needed.

\section{Catheter Angiography}

Conventional angiography remains the imaging criterion standard for the evaluation of cerebral vasculature and may detect cerebral vasoconstriction in patients whose initial noninvasive vascular imaging findings appear unremarkable. ${ }^{12}$ This is particularly true in the evaluation of small, distal cortical vessels, which are suboptimally evaluated by CTA or MRA secondary to their inferior spatial resolution. Ducros and Bousser ${ }^{2}$ found that noninvasive imaging with MRA and CTA demonstrated sensitivity for detecting RCVS-vasoconstriction of $80 \%$ compared with conventional angiography. In our experience, conventional angiography has been proved an invaluable tool when clinical diagnosis is equivocal and noninvasive imaging findings are normal. For example, it may help evaluate patients with sus- 


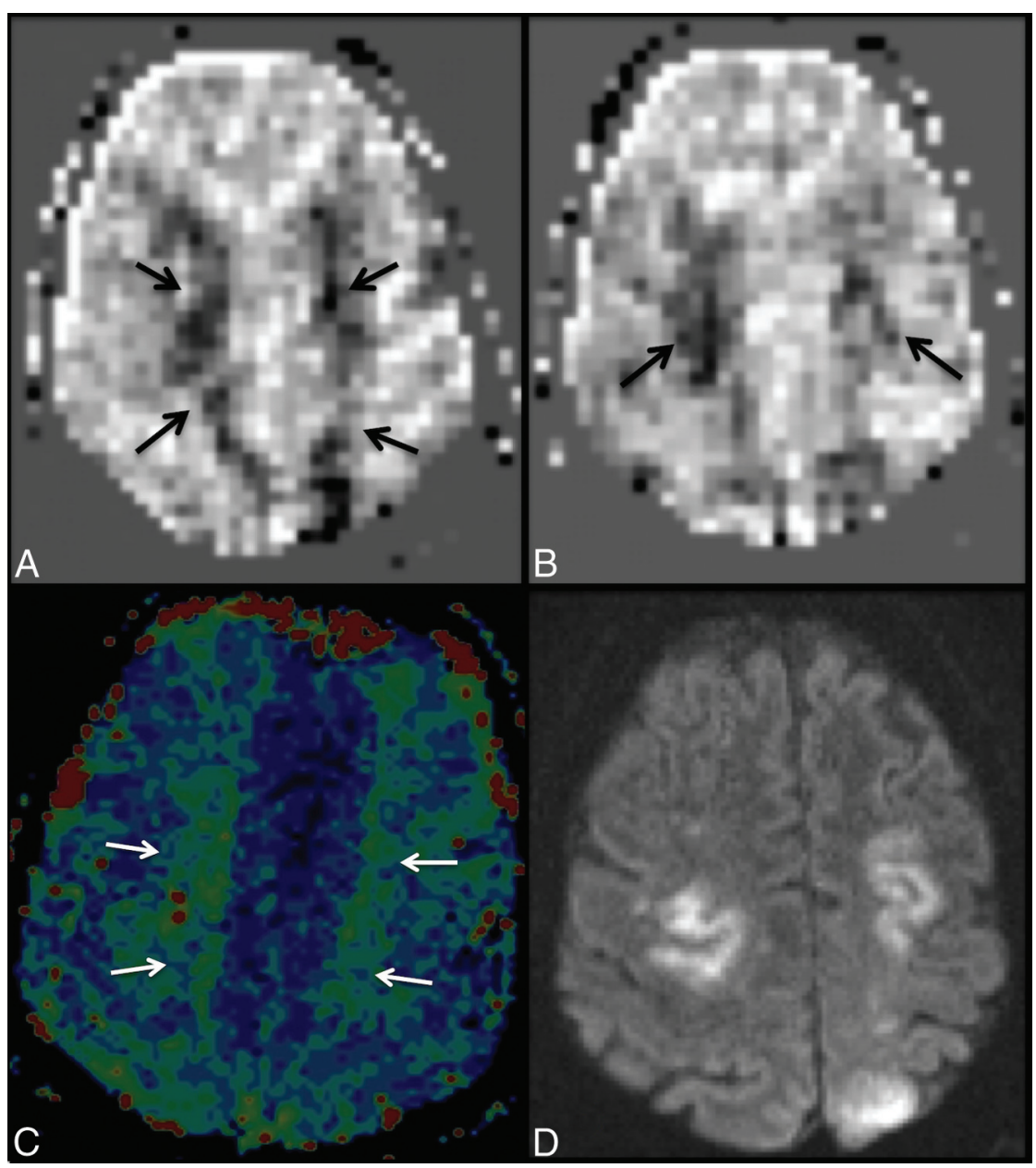

FIG 3. A 55-year-old woman (the same patient as in Fig 1) with RCVS complicated by ischemic infarcts. Axial CBF pulsed arterial spin-labeling maps $(A$ and $B$ ) show multiple regions of diminished perfusion involving anterior cerebral artery/MCA watershed territories (black arrows), with T2* DSC perfusion time-to-peak maps (C) demonstrating delayed time-to-peak in these same regions (white arrows). These areas of perfusion abnormality correspond, in part, to regions of restriction diffusion/ischemic infarct on DWI (D).

pected RCVS who either present in a somewhat atypical fashion (eg, more insidious-onset headache, no obvious risk factors) or demonstrate a plausible alternative diagnosis (eg, cerebral aneurysm arising from the circle of Willis). In these instances, better visualization of the character and distribution of cerebral artery irregularity and the morphology of any cerebral aneurysms present can be helpful.

Additionally, DSA may provide complementary information to aid the diagnosis, including reversibility of vasoconstriction following intra-arterial administration of a vasodilator. ${ }^{48-52} \mathrm{Be}-$ cause diagnostic confirmation of RCVS is usually retrospective following spontaneous resolution of clinical and angiographic findings in 1-3 months, there is often a substantial delay in confirming the diagnosis. Consequently, demonstration of reversibility following intra-arterial vasodilator administration can be clinically useful in the early recognition of RCVS, as opposed to the partial or incomplete improvement often seen with other vasospastic disorders. ${ }^{52}$ At this time, the potential risks of such a diagnostic challenge remain uncertain and perhaps may be unnecessary if the clinical and radiologic findings are otherwise supportive of a diagnosis of RCVS.
Although conventional angiography is generally a safe procedure when performed by experienced operators, a suggestion has been made that there may be an increased risk of transient ischemic attack in patients with RCVS. Ducros et $\mathrm{al}^{12}$ reported a $9 \%$ incidence of new transient neurologic deficits within 1 hour following conventional angiography in 67 patients. However, Katz et al, ${ }^{53}$ in their retrospective study, failed to demonstrate a similar increased risk of clinical deterioration in patients with RCVS undergoing conventional angiography. In our experience, both published and unpublished, we have found no increase in complication rates following cerebral angiography for RCVS. ${ }^{10}$

\section{Differential Diagnosis}

As previously discussed, presenting symptoms, sequelae, and radiographic features of RCVS can significantly overlap other frequently encountered medical conditions involving the CNS (Table 2). ${ }^{1,2,7,15,21,23}$ Furthermore, treatment of some of these alternative diagnoses, including aneurysmal subarachnoid hemorrhage and PACNS, varies considerably from that of RCVS, making an accurate diagnosis critical to ensuring appropriate patient care. ${ }^{13,19}$ The following section will highlight important clinical and radiologic findings that can help differentiate some of these entities from RCVS.

Cerebral Aneurysm Rupture with Subarachnoid Hemorrhage. Differentiating RCVS from aneurysmal subarachnoid hemorrhage can be challenging due to the overlap in patient symptomatology and radiographic features. ${ }^{54}$ In particular, the scenario of thunderclap headache, sulcal subarachnoid hemorrhage, and a remote cerebral aneurysm at or near the circle of Willis can be particularly difficult. ${ }^{55}$ As previously discussed, the relapsingremitting thunderclap headache typical of RCVS would be highly unusual for patients with aneurysmal subarachnoid hemorrhage. ${ }^{1,2,11,12,21,29}$ Although aneurysmal subarachnoid hemorrhage is overall the most common cause of nonprimary thunderclap headache, RCVS is the most probable diagnosis in patients who experience episodic thunderclap headaches for 1-2 weeks. $^{55}$

Furthermore, patients with aneurysmal subarachnoid hemorrhage often demonstrate acute, progressive neurologic decline following presentation due to complications such as increased intracranial pressure and communicating hydrocephalus, which, again, would be atypical for RCVS. A retrospective analysis of patients with RCVS $(n=38)$, aneurysmal subarachnoid hemor- 
rhage $(n=515)$, and cryptogenic subarachnoid hemorrhage $(n=$ 93) by Muehlschlegel et $\mathrm{al}^{56}$ found that among other factors, younger patient age, less severe neurologic symptoms, and better clinical grade (ie, lower Hunt and Hess scale score) were predictive of RCVS as opposed to aneurysmal subarachnoid hemorrhage. However, one clinical scenario that could more closely mimic RCVS would be a patient experiencing a small sentinel hemorrhage from a cerebral aneurysm, which could produce a similar clinical course with waxing and waning symptoms. ${ }^{55}$

Imaging can also help differentiate RCVS from aneurysmal (or perimesencephalic) subarachnoid hemorrhage. First, many patients with RCVS will have unremarkable findings on a noncontrast head CT examination, without evidence of intracranial hemorrhage or infarct. ${ }^{2}$ In cases of RCVS complicated by intracranial hemorrhage, the pattern has focal subarachnoid hemorrhage most often confined to superficial cerebral sulci, which is in contradistinction to aneurysmal subarachnoid hemorrhage, in which blood is most often centered at the basal cisterns/circle of Willis. ${ }^{2,5,10,15,16}$ This pattern of subarachnoid hemorrhage may also help differentiate RCVS from nonaneurysmal subarachnoid hemorrhage on angiography, which typically predominate in the perimesencephalic region. ${ }^{16}$

In patients who present with thunderclap headache and localized, sulcal subarachnoid hemorrhage, the presence of a cerebral aneurysm arising more proximally near the circle of Willis can pose a diagnostic challenge. ${ }^{15}$ In these instances, evaluating the patient's clinical course and symptomatology may help differentiate the 2 diagnoses. VWI may be useful in these instances by evaluating the aneurysm for wall enhancement, which would suggest inflammation and possible recent rupture. However, the validity of this technique remains uncertain.

Differentiating RCVS cerebral vasoconstriction from arterial vasospasm associated with aneurysmal subarachnoid hemorrhage can also be difficult. On the basis of their clinical experience

Table 2: Potential alternative diagnoses

Aneurysmal SAH
Primary angiitis of the CNS
Migraine
Cortical vein thrombosis
Pituitary apoplexy
Amyloid angiopathy
Hypertensive hemorrhage
PRES
Giant cell arteritis
Arterial dissection
Spontaneous intracranial hypotension
Meningitis

Note:-PRES indicates posterior reversible encephalopathy syndrome. and review of the literature, Ansari et $\mathrm{al}^{10}$ suggested several diagnostic criteria to help differentiate these 2 entities, focusing on the severity, distribution, and time of onset of cerebral artery narrowing and the relation of these findings to a potential culprit aneurysm (Table 3). Unfortunately, none of these diagnostic criteria, either alone or in combination, are entirely specific for RCVS vasoconstriction or arterial vasospasm. For example, although RCVS vasoconstriction is often noted to involve distal cerebral arteries, more proximal vessel involvement occurs. In addition, the delay in the appearance of RCVS vasoconstriction may mimic the typical time course of arterial vasospasm. Conversely, hyperacute vasospasm may occasionally be associated with aneurysmal subarachnoid hemorrhage. ${ }^{15}$ Consequently, considering the patient's overall clinical picture and radiographic features may be the most effective way of differentiating these 2 entities.

Primary Angiitis of the CNS. Although differentiating severe RCVS and PACNS can be challenging because the 2 entities overlap in clinical and radiographic features, the distinction is critical because treatment significantly differs. ${ }^{2,7,13}$ Patients with PACNS often experience a fulminant course with a poor prognosis if immunosuppressive therapy with steroids and cytotoxic agents is not initiated early, while these medications are not beneficial in patients with RCVS and may be harmful. ${ }^{1,2,13,14}$ Fortunately, a correct diagnosis can be made in most patients by considering multiple factors, including the onset and severity of patient symptoms, patient demographics, CSF and imaging findings, and specific disease sequelae.

The headache associated with PACNS is often slowly progressive with an insidious onset, differing markedly from the typical thunderclap headache of RCVS in both time course and peak severity. ${ }^{1,2,7,13,16,18,19,57}$ Patient demographics in these disease entities also demonstrate significant differences. RCVS is typically encountered in young-to-middle-aged women, as opposed to PACNS, which is most often seen in older men. ${ }^{14,18,19}$ Analysis of CSF is also helpful because patients with PACNS, in contradistinction to RCVS, typically demonstrate elevations of CSF protein levels and white blood cell count, with values often $>100 \mathrm{mg} / \mathrm{dL}$ and $5-10$ cells/mm, respectively. ${ }^{1,2,7,10,16,18}$ Finally, the early clinical course of the patient can help distinguish these 2 entities. RCVS generally follows a benign, self-limited course with supportive care, while clinical deterioration would be expected in PACNS without prompt immunosuppressive therapy. ${ }^{2,18}$

Nonvascular imaging findings can also help differentiate PACNS and RCVS. Most patients with PACNS will demonstrate evidence of multifocal infarcts of varying ages on presentation (90\%), compared with initial MR imaging findings in patients with RCVS, which are often unremarkable. ${ }^{2,18}$ This finding is

Table 3: Proposed criteria for differentiating RCVS vasoconstriction from SAH vasospasm ${ }^{10}$

\begin{tabular}{ll}
\hline \multicolumn{1}{c}{ RCVS Vasoconstriction } & Vasospasm-Aneurysmal SAH \\
\hline $\begin{array}{l}\text { No evidence of ruptured aneurysm or vascular malformation } \\
\text { Diffuse and disproportionate extent of cerebral vasoconstriction } \\
\text { relative to amount of SAH }\end{array}$ & $\begin{array}{c}\text { Plausible target lesion identified } \\
\text { Severity of vasospasm correlates with amount of hemorrhage } \\
\text { and is most pronounced in the vicinity of the lesion } \\
\text { preferentially involving distal 2nd- and 3rd-order cerebral } \\
\text { branches }\end{array}$ \\
$\begin{array}{c}\text { Smooth, long segmental narrowing for proximal arteries at } \\
\text { circle of Willis } \\
\text { onset, or persistence past 3 weeks }\end{array}$ & $\begin{array}{c}\text { Development of vasospasm peaking between } 4 \text { and 14 days } \\
\text { after hemorrhage }\end{array}$ \\
\hline
\end{tabular}




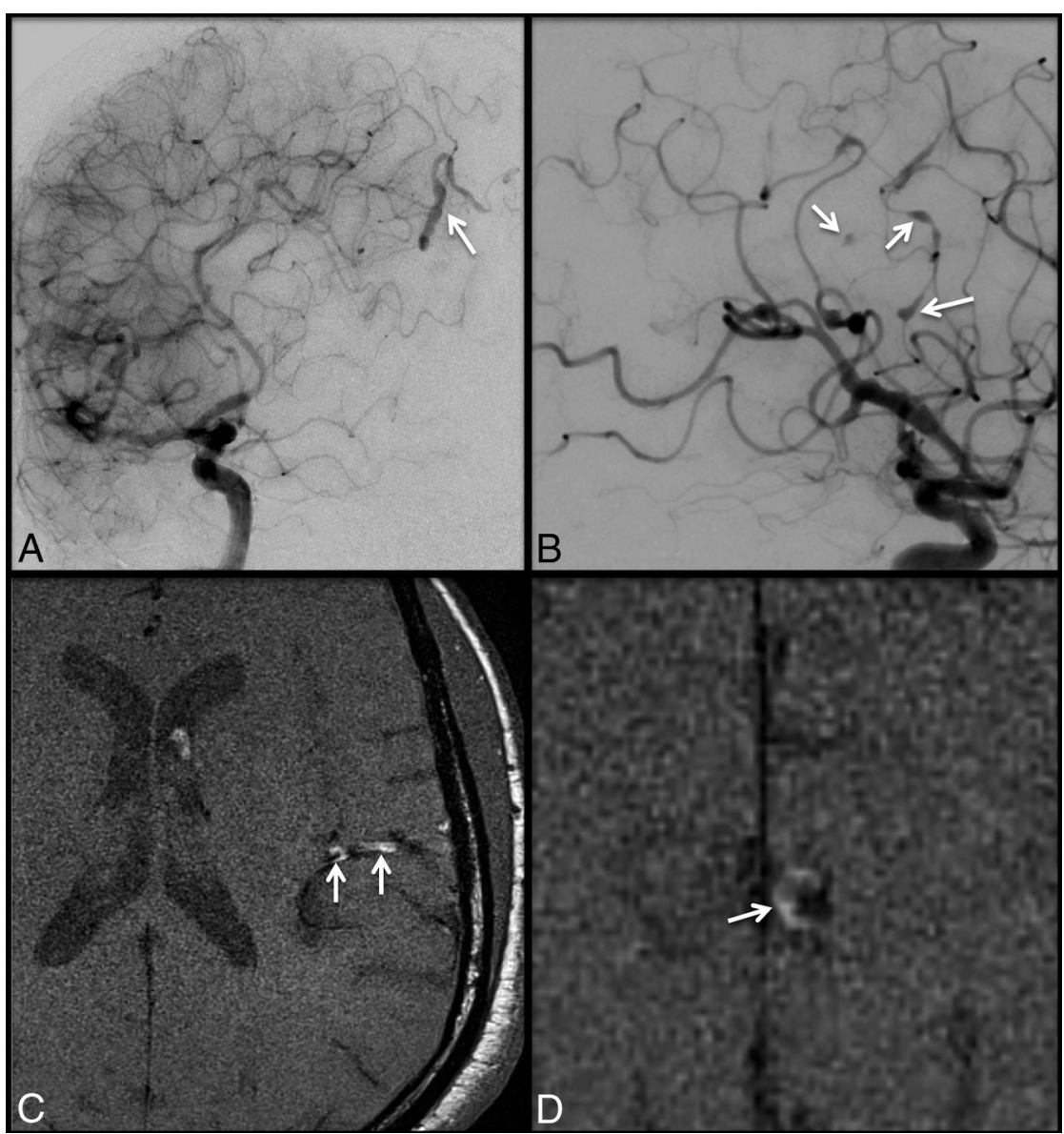

FIG 4. A 59-year-old man with a history of seizures, who was subsequently found to have multifocal infarcts in several vascular territories (not shown). Subsequent catheter angiograms ( $A$ and $B$ ) demonstrate marked irregularity of branches of the distal right anterior cerebral artery (white arrow, $A$ ) and left MCA (white arrows, B), with multifocal areas of narrowing and saccular and fusiform dilation. On axial T1 precontrast high-resolution VWI $(C)$, there is intrinsic T1 mural hyperintensity in involved MCA (white arrows) and anterior cerebral artery branches. On axial T1 postcontrast high-resolution VWI (D), there are accompanying areas of eccentric vessel wall enhancement (white arrow, D).

consistent with the later timeframe during which ischemic stroke typically occurs in the course of RCVS, as previously discussed. ${ }^{12}$ Hemorrhagic complications, including cortical subarachnoid hemorrhage and concomitant posterior reversible encephalopathy syndrome, which are well-established features of RCVS, are extremely unusual in cases of PACNS. ${ }^{1,14,18}$

Imaging of the cerebral vasculature can also assist in the diagnostic work-up. Although PACNS can produce a pattern of multifocal narrowing and irregularity of mid-to-distal cerebral arteries that is indistinguishable from RCVS, most cases will appear unremarkable on angiographic imaging. ${ }^{218-20}$ This appearance is true even for the criterion standard of conventional angiography, which has a reported sensitivity of only $20 \%-64 \%$ for detecting CNS vasculitis. ${ }^{7,10,29}$ Alternatively, cerebral vasoconstriction is often apparent in cases of RCVS at presentation or shortly thereafter. Some authors have argued that certain angiographic features are more characteristic of PACNS, including eccentric luminal narrowing and abrupt vessel occlusions (Fig 4) ${ }^{2,7,10}$ However, the specificity of these findings for PACNS remains uncertain. Finally, improvement in cerebral artery narrowing following in- tra-arterial vasodilator therapy has also been proposed as a feature distinguishing RCVS from PACNS. ${ }^{52}$

Cortical Vein Thrombosis. Cortical vein thrombosis is another potential cause of both thunderclap headache and convexity subarachnoid hemorrhage and should be considered in the differential diagnosis with RCVS in the appropriate clinical setting. Postpartum women are one specific subgroup of patients who are at increased risk for both disease entities. ${ }^{1} \mathrm{MR}$ imaging, including susceptibility sequences and MRV, can provide high specificity for the diagnosis of cortical vein thrombosis. MR imaging can demonstrate characteristic susceptibility artifacts associated with a superficial cortical vein consistent with thrombus. Both RCVS and cortical vein thrombosis can lead to ischemic stroke, often a week or more after the onset of symptoms. As is the case with primary angiitis of the CNS, distinguishing RCVS from cortical vein thrombosis is critical because treatment of the latter often entails anticoagulation, which carries significant risks and has not been shown to be beneficial in RCVS.

Migraine Headache and Stroke. The association between migraine headache and RCVS can make differentiating these 2 entities challenging. ${ }^{1,5,16}$ Both entities can present with thunderclap headache, associated photo- and phonophobia, and nausea and vomiting. ${ }^{7}$

Furthermore, migrainous headaches have rarely even been associated with ischemic stroke. However, most patients with a history of migraine who present with RCVS describe the quality and severity of the pain as being different from that in their typical migraine. ${ }^{7,15}$ Ischemic stroke in patients with migraine tends to be limited to a single vascular territory, as opposed to RCVS, in which multiterritory involvement is common. ${ }^{7}$

Amyloid Angiography. Although both amyloid angiopathy and RCVS can result in lobar intraparenchymal hematoma and cortical subarachnoid hemorrhage, amyloid angiography is encountered in older individuals and typically does not present with a thunderclap or acute-onset headache. ${ }^{1,58}$ Kumar et al ${ }^{58}$ retrospectively evaluated a group of patients with atraumatic convexity subarachnoid hemorrhage and found 2 distinct patterns of clinical presentation. In patients younger than 60 years of age, presentation with abrupt, severe headache was common, and most of these individuals were presumptively diagnosed with RCVS. In contradistinction, patients older than 60 years of age most commonly presented with transitory neurologic deficits and had evi- 
dence of leukoaraiosis and microhemorrhages on MR imaging. Most of these patients were diagnosed with cerebral amyloid angiopathy.

\section{CONCLUSIONS}

Imaging plays a critical role in the diagnosis and management of RCVS. Noninvasive techniques such as MR angiography are being increasingly used in clinical practice, though cerebral angiography remains the criterion standard for the detection of cerebral vasoconstriction. Clinical and imaging features of RCVS can overlap other disorders of the central nervous system considerably, particularly primary angiitis of the CNS. However, newer imaging techniques, particularly vessel wall imaging, may offer increased specificity for the diagnosis.

\section{REFERENCES}

1. Ducros A. L37: reversible cerebral vasoconstriction syndromedistinction from CNS vasculitis. Presse Med 2013;42(4 pt 2):602-04

2. Ducros A, Bousser MG. Reversible cerebral vasoconstriction syndrome. Pract Neurol 2009;9:256-67

3. Gupta S, Zivadinov R, Ramasamy D, et al. Reversible cerebral vasoconstriction syndrome (RCVS) in antiphospholipid antibody syndrome (APLA): the role of centrally acting vasodilators-case series and review of literature. Clin Rheumatol 2014;33:1829-33

4. Marder CP, Donohue MM, Weinstein JR, et al. Multimodal imaging of reversible cerebral vasoconstriction syndrome: a series of 6 cases. AJNR Am J Neuroradiol 2012;33:1403-10

5. Sheikh HU, Mathew PG. Reversible cerebral vasoconstriction syndrome: updates and new perspectives. Curr Pain Headache Rep 2014; $18: 414$

6. Stary JM, Wang BH, Moon SJ, et al. Dramatic intracerebral hemorrhagic presentations of reversible cerebral vasoconstriction syndrome: three cases and a literature review. Case Rep Neurol Med 2014;2014:782028

7. Calabrese LH, Dodick DW, Schwedt TJ, et al. Narrative review: reversible cerebral vasoconstriction syndromes. Ann Intern Med 2007;146:34-44

8. Lin CH, Chen YY, Chiu LA, et al. Dual energy computed tomography angiography for the rapid diagnosis of reversible cerebral vasoconstriction syndromes: report of a case. Acta Neurol Taiwan 2013;22:36-42

9. Singhal AB, Bernstein RA. Postpartum angiopathy and other cerebral vasoconstriction syndromes. Neurocrit Care 2005;3:91-97

10. Ansari SA, Rath TJ, Gandhi D. Reversible cerebral vasoconstriction syndromes presenting with subarachnoid hemorrhage: a case series. J Neurointerv Surg 2011;3:272-78

11. Bain J, Segal D, Amin R, et al. Call-Fleming syndrome: headache in a 16-year-old girl. Pediatr Neurol 2013;49:130-33.e1

12. Ducros A, Boukobza M, Porcher $\mathrm{R}$, et al. The clinical and radiological spectrum of reversible cerebral vasoconstriction syndrome: a prospective series of 67 patients. Brain 2007;130(pt 12):3091-101

13. Hajj-Ali RA, Furlan A, Abou-Chebel A, et al. Benign angiopathy of the central nervous system: cohort of 16 patients with clinical course and long-term followup. Arthritis Rheum 2002;47:662-69

14. Hammad TA, Hajj-Ali RA. Primary angiitis of the central nervous system and reversible cerebral vasoconstriction syndrome. Curr Atheroscler Rep 2013;15:346

15. Edlow BL, Kasner SE, Hurst RW, et al. Reversible cerebral vasoconstriction syndrome associated with subarachnoid hemorrhage. Neurocrit Care 2007;7:203-10

16. Singhal AB, Hajj-Ali RA, Topcuoglu MA, et al. Reversible cerebral vasoconstriction syndromes: analysis of 139 cases. Arch Neurol 2011;68:1005-12

17. Ducros A. Reversible cerebral vasoconstriction syndrome. Lancet Neurol 2012;11:906-17
18. Hajj-Ali RA, Singhal AB, Benseler S, et al. Primary angiitis of the CNS. Lancet Neurol 2011;10:561-72

19. Koopman K, Uyttenboogaart M, Luijckx GJ, et al. Pitfalls in the diagnosis of reversible cerebral vasoconstriction syndrome and primary angiitis of the central nervous system. Eur J Neurol 2007; 14:1085-87

20. Obusez EC, Hui F, Hajj-Ali RA, et al. High-resolution MRI vessel wall imaging: spatial and temporal patterns of reversible cerebral vasoconstriction syndrome and central nervous system vasculitis. AJNR Am J Neuroradiol 2014;35:1527-32

21. Grooters GS, Sluzewski M, Tijssen CC. How often is thunderclap headache caused by the reversible cerebral vasoconstriction syndrome? Headache 2014;54:732-35

22. Chen SP, Wang SJ. Hyperintense vessels: an early MRI marker of reversible cerebral vasoconstriction syndrome? Cephalalgia 2014; 34:1038-39

23. Calic Z, Choong H, Schlaphoff G, et al. Reversible cerebral vasoconstriction syndrome following indomethacin. Cephalalgia 2014;34: 1181-86

24. Chen SP, Fuh JL, Chang FC, et al. Transcranial color Doppler study for reversible cerebral vasoconstriction syndromes. Ann Neurol 2008;63:751-57

25. Koopman K, Teune LK, ter Laan M, et al. An often unrecognized cause of thunderclap headache: reversible cerebral vasoconstriction syndrome. J Headache Pain 2008;9:389-91

26. Chen SP, Fuh JL, Lirng JF, et al. Hyperintense vessels on FLAIR imaging in reversible cerebral vasoconstriction syndrome. Cephalalgia 2012;32:271-78

27. Iancu-Gontard D, Oppenheim C, Touze E, et al. Evaluation of hyperintense vessels on FLAIR MRI for the diagnosis of multiple intracerebral arterial stenoses. Stroke 2003;34:1886-91

28. Kameda T, Namekawa M, Shimazaki H, et al. Unique combination of hyperintense vessel sign on initial FLAIR and delayed vasoconstriction on MRA in reversible cerebral vasoconstriction syndrome: a case report. Cephalalgia 2014;34:1093-96

29. Chen SP, Fuh JL, Wang SJ, et al. Magnetic resonance angiography in reversible cerebral vasoconstriction syndromes. Ann Neurol 2010;67:648-56

30. Mandell DM, Matouk CC, Farb RI, et al. Vessel wall MRI to differentiate between reversible cerebral vasoconstriction syndrome and central nervous system vasculitis: preliminary results. Stroke 2012;43:860-62

31. Küker W, Gaertner S, Nagele T, et al. Vessel wall contrast enhancement: a diagnostic sign of cerebral vasculitis. Cerebrovasc Dis 2008;26:23-29

32. Swartz RH, Bhuta SS, Farb RI, et al. Intracranial arterial wall imaging using high-resolution 3-Tesla contrast-enhanced MRI. Neurology 2009;72:627-34

33. Ryoo S, Cha J, Kim SJ, et al. High-resolution magnetic resonance wall imaging findings of Moyamoya disease. Stroke 2014;45:2457-60

34. Kim YJ, Lee DH, Kwon JY, et al. High resolution MRI difference between Moyamoya disease and intracranial atherosclerosis. Eur J Neurol 2013;20:1311-18

35. Qiao Y, Steinman DA, Qin Q, et al. Intracranial arterial wall imaging using three-dimensional high isotropic resolution black blood MRI at 3.0 Tesla. J Magn Reson Imaging 2011;34:22-30

36. Natori T, Sasaki M, Miyoshi M, et al. Evaluating middle cerebral artery atherosclerotic lesions in acute ischemic stroke using magnetic resonance T1-weighted 3-dimensional vessel wall imaging. $J$ Stroke Cerebrovasc Dis 2014;23:706-11

37. van der Kolk AG, Zwanenburg JJ, Brundel M, et al. Intracranial vessel wall imaging at 7.0-T MRI. Stroke 2011;42:2478-84

38. Qiao Y, Zeiler SR, Mirbagheri S, et al. Intracranial plaque enhancement in patients with cerebrovascular events on high-spatial-resolution MR images. Radiology 2014;271:534-42

39. Degnan AJ, Gallagher G, Teng Z, et al. MR angiography and imaging 
for the evaluation of middle cerebral artery atherosclerotic disease. AJNR Am J Neuroradiol 2012;33:1427-35

40. $\mathrm{Xu} \mathrm{WH,} \mathrm{Li} \mathrm{ML,} \mathrm{Gao} \mathrm{S,} \mathrm{et} \mathrm{al.} \mathrm{In} \mathrm{vivo} \mathrm{high-resolution} \mathrm{MR} \mathrm{imaging} \mathrm{of}$ symptomatic and asymptomatic middle cerebral artery atherosclerotic stenosis. Atherosclerosis 2010;212:507-11

41. Niizuma K, Shimizu H, Takada S, et al. Middle cerebral artery plaque imaging using 3-Tesla high-resolution MRI. J Clin Neurosci 2008; 15:1137-41

42. Kamath $\mathrm{S}$. Observations on the length and diameter of vessels forming the circle of Willis. J Anat 1981;133(pt 3):419-23

43. $\mathrm{Xu} \mathrm{WH,} \mathrm{Li} \mathrm{ML,} \mathrm{Gao} \mathrm{S,} \mathrm{et} \mathrm{al.} \mathrm{Middle} \mathrm{cerebral} \mathrm{artery} \mathrm{intraplaque}$ hemorrhage: prevalence and clinical relevance. Ann Neurol 2012; 71:195-98

44. Hochberg AR, Young GS. Cerebral perfusion imaging. Semin Neurol 2012;32:454-65

45. Telischak NA, Detre JA, Zaharchuk G. Arterial spin labeling MRI: clinical applications in the brain. J Magn Reson Imaging 2014 Sep 19. [Epub ahead of print]

46. Komatsu T, Kimura T, Yagishita A, et al. A case of reversible cerebral vasoconstriction syndrome presenting with recurrent neurological deficits: evaluation using noninvasive arterial spin labeling MRI. Clin Neurol Neurosurg 2014;126:96-98

47. Rosenbloom MH, Singhal AB. CT angiography and diffusion-perfusion MR imaging in a patient with ipsilateral reversible cerebral vasoconstriction after carotid endarterectomy. AJNR Am J Neuroradiol 2007;28:920-22

48. Ioannidis I, Nasis N, Agianniotaki A, et al. Reversible cerebral vasoconstriction syndrome: treatment with multiple sessions of intraarterial nimodipine and angioplasty. Interv Neuroradiol 2012;18: 297-302

49. French KF, Hoesch RE, Allred J, et al. Repetitive use of intra-arterial verapamil in the treatment of reversible cerebral vasoconstriction syndrome. J Clin Neurosci 2012;19:174-76

50. Farid H, Tatum JK, Wong C, et al. Reversible cerebral vasoconstriction syndrome: treatment with combined intra-arterial verapamil infusion and intracranial angioplasty. AJNR Am J Neuroradiol 2011;32:E184-87

51. Elstner M, Linn J, Muller-Schunk S, et al. Reversible cerebral vasoconstriction syndrome: a complicated clinical course treated with intra-arterial application of nimodipine. Cephalalgia 2009;29:677-82

52. Linn J, Fesl G, Ottomeyer C, et al. Intra-arterial application of nimodipine in reversible cerebral vasoconstriction syndrome: a diagnostic tool in select cases? Cephalalgia 2011;31:1074-81

53. Katz BS, Fugate JE, Ameriso SF, et al. Clinical worsening in reversible cerebral vasoconstriction syndrome. JAMA Neurol 2014;71:68-73

54. Nickele C, Muro K, Getch CC, et al. Severe reversible cerebral vasoconstriction syndrome mimicking aneurysmal rupture and vasospasm. Neurocrit Care 2007;7:81-85

55. Chen SP, Fuh JL, Wang SJ. Reversible cerebral vasoconstriction syndrome: current and future perspectives. Expert Rev Neurother 2011;11:1265-76

56. Muehlschlegel S, Kursun O, Topcuoglu MA, et al. Differentiating reversible cerebral vasoconstriction syndrome with subarachnoid hemorrhage from other causes of subarachnoid hemorrhage. JAMA Neurol 2013;70:1254-60

57. Nouh A, Ruland S, Schneck MJ, et al. Reversible cerebral vasoconstriction syndrome with multivessel cervical artery dissections and a double aortic arch. J Stroke Cerebrovasc Dis 2014;23:e141-43

58. Kumar S, Goddeau RP Jr, Selim MH, et al. Atraumatic convexal subarachnoid hemorrhage: clinical presentation, imaging patterns, and etiologies. Neurology 2010;74:893-99 